\section{SAT0670 THE PREVALENCE OF NEUROPATHIC PAIN-LIKE SYMPTOMS AND ASSOCIATED RISK FACTORS IN THE NOTTINGHAM COMMUNITY: A CROSS-SECTIONAL STUDY}

G.S. Fernandes ${ }^{1,2,3}$, A. Sarmanova ${ }^{1,3}$, S. Warner ${ }^{1}$, H. Harvey ${ }^{1}$ H. Richardson ${ }^{1}$, N. Frowd ${ }^{1,3}$, L. Marshall ${ }^{1,3}$, J. Stocks ${ }^{1,3}$, M. Hall 1,3 A. Valdes ${ }^{1,2,3}$, D. Walsh ${ }^{1,2,3}$, W. Zhang ${ }^{1,2,3}$, M. Doherty ${ }^{1,2,3}$. ${ }^{1}$ Division of Rheumatology, Orthopaedics and Dermatology; ${ }^{2}$ Arthritis Research UK Centre for Sports, Exercise and Osteoarthritis; ${ }^{3}$ Arthritis Research UK Pain Centre, University of Nottingham, Nottingham, United Kingdom

Background: Knee pain (KP) affects 1 in 4 adults over the age of 50 . Aside from structural joint changes, person-specific factors influence the KP experience. Increased central sensitisation of neural pathways due to localised joint pain or ineffective descending inhibitory mechanisms can cause an enhanced pain response and neuropathic pain-like (NP) symptoms. Understanding these personspecific factors and how they modulate the pain experience might help profile different KP and NP phenotypes.

Objectives: a) To determine the prevalence of NP in a KP community population b) to identify significant risk factors associated with NP and those with both NP and non-NP KP.

Methods: 9,513 men and women, aged 40+ years, were recruited from the East Midlands region (United Kingdom) via postal questionnaire. The questionnaire included sections on KP severity (numerical rating scale) and type (NP versus nociceptive) using the modified PainDETECT Questionnaire (mPDQ); quality of $\mathrm{KP}$ using the intermittent and constant osteoarthritis pain (ICOAP) instrument) as well as other risk factors including age, body mass index (BMI), injury, pain catastrophizing scale (PCS) and mental wellness (Hospital Anxiety and Depression Scale). KP participants were those who reported "knee pain for most days of the past one month" while likely NP was MPDQ scores of $\geq 13$ and definite $N P, \geq 19$. Differences between groups were assessed using $t$-tests for continuous data and $\mathrm{chi}^{2}$ for categorical data. We used multinomial regression analysis to determine the odds ratios (ORs) of risk factors with $95 \%$ confidence intervals $(\mathrm{Cl})$ and significance set $p<0.05$.

Results: The prevalence of definite NP in the Nottingham Community was 366 $(13.62 \%)$. There were more women $(\mathrm{p}=0.04)$ and higher $\mathrm{BMI}(\mathrm{p}<0.001)$ in $\mathrm{KP}$ vs. non-KP responders but no age difference $(p>0.05)$. When comparing the neuropathic-like KP to non-neuropathic KP responders, significant risk factors after adjustment for age, BMI, gender and pain severity included: anxiety (OR 3.17 (95\% Cl 2.38;4.23)); depression (OR 2.99 (95\% Cl 2.14;4.19)); PCS in highest tertile (OR $5.39(95 \% \mathrm{Cl} 2.94 ; 9.88))$; fibromyalgia (OR $4.06(95 \%$ $\mathrm{Cl} 2.48 ; 6.66)$ ) and previous knee injury (OR $1.5(95 \% \mathrm{Cl} 1.12 ; 2.00))$. When comparing neuropathic-like KP to non-KP responders, anxiety $(1.74(95 \% \mathrm{Cl}$ $1.31 ; 2.30)$, depression $(2.05(95 \% \mathrm{Cl} 1.40 ; 3.01)$, PCS 3.78 (95\% Cl $2.57 ; 5.56)$ ), fibromyalgia (OR $1.94(95 \% \mathrm{Cl} 1.10 ; 3.40)$ ) and previous injury (OR $1.35(95 \% \mathrm{Cl}$ $1.05 ; 1.73)$ ) were significant risk factors after adjustment.

Conclusions: This is the first population based cross-sectional study in the UK to determine prevalence of NP in people with KP. The results suggest that both psychological factors (depression, anxiety, high catastrophising) and peripheral risk factors (injury) associate with NP reporting. These factors can augment pain sensitivity and produce an amplified response via central and peripheral pathways. Phenotypes based on these risk factor profiles may warrant specific management in KP populations.

Acknowledgements: Arthritis Research UK Grant Refs: 20777, 20194

Disclosure of Interest: None declared

DOI: 10.1136/annrheumdis-2017-eular.4930

\section{SAT0671 THE IMPACT OF OBESITY ON TREAT TO TARGET GOALS AND FUNCTIONAL ABILITY IN THE ERAS/ERAN UK PROSPECTIVE COHORTS}

E. Nikiphorou ${ }^{1}$, S. Norton ${ }^{1}$, P. Kiely ${ }^{2}$, A. Young ${ }^{3}$ on behalf of ERAS/ERAN. ${ }^{1} K C L ;{ }^{2} S t$ George's Hospital, London; ${ }^{3}$ St Albans Hospital, St Albans, United Kingdom

Background: The links between adipose tissue and inflammation on the one hand and obesity and joint dysfunction on the other, are well established. However, how these translate into clinical disease activity and functional disability in rheumatoid arthritis (RA), remains to be clearly defined.

Objectives: To investigate the association between $\mathrm{BMI}$ and 1. The achievement of disease remission or low disease activity and 2. Functional ability, in RA.

Methods: Data from two consecutive UK multi-centre RA inception cohorts with similar design were used: the Early RA Study (ERAS) and Early RA Network (ERAN). Recruitment figures/median follow up for ERAS and ERAN were 1465/10 years (maximum 25 years), and 1236/6 years (maximum 10 years) respectively. Standard demographic and clinical variables were recorded at baseline and then annually until loss to follow-up or the end of study follow-up. Multilevel logistic regression analysis was used with either remission (R-DAS) or low disease activity status (L-DAS) and health assessment questionnaire (HAQ, $<1$ vs $>1$ ) as the dependent categorical variables of interest in models adjusting for patient, disease-related clinical variables and recruitment year. BMI was examined in separate models as both a continuous and categorical predictor variable according to WHO definitions: underweight (BMI less than 18.5), normal (BMI between 18.5 and 25), overweight (BMI between 25 and 30 ) and obese (BMI greater than 30 ).
BMI was included in the models relating to the same time point as the outcome assessed.

Results: Baseline BMI data from 2420 patients (90\%) indicated that $40.0 \%$ had BMl scores in the normal range, $1.8 \%$ were underweight, $37.2 \%$ were overweight and $21.3 \%$ were obese. Mean BMI increased slightly over time from 26.5 at baseline to 26.8 at 2 years and then 27.1 at 5 years. In multilevel logistic models adjusting for age, sex, smoking status, antibody status, haemoglobin, erosions and year of recruitment, higher BMI was associated with reduced odds of achieving R-DAS (OR $0.97 ; 95 \% \mathrm{Cl} 0.95,0.99$ ) (table) and L-DAS, although the latter did not reach statistical significance (OR $0.98 ; 95 \% \mathrm{Cl} 0.96,1.00)$. Obesity was related to a significantly lower chance of R-DAS by $29 \%$ (OR $0.71 ; 95 \% \mathrm{Cl} 0.55,0.93$ ) and L-DAS by $31 \%(\mathrm{OR} 0.69 ; 95 \% \mathrm{Cl} 0.55,0.87)$. Higher $\mathrm{BMI}$ was predictive of higher disability (OR $1.04 ; 95 \% \mathrm{Cl} 1.01,1.06)$. More specifically, obesity increased the odds of higher disability by $63 \%$ (OR $1.63 ; 95 \% \mathrm{Cl} 1.20,2.23)$ and in the same models, higher DAS was also strongly predictive of higher disability (OR $3.67 ; 95 \%$ $\mathrm{Cl} 3.41,3.95)$

Table. Impact of BMI category on disease activity and functional ability in models adjusting for patient demographic, clinical \& disease variables.

\begin{tabular}{|c|c|c|c|}
\hline \multirow[b]{2}{*}{ Independent predictors } & \multicolumn{3}{|c|}{ MODELS } \\
\hline & $\begin{array}{l}\text { Remission DAS } \\
\text { OR }(95 \% \text { CI }) \\
(n=1690)\end{array}$ & $\begin{array}{l}\text { Low DAS } \\
\text { OR }(95 \% \mathrm{CI}) \\
(\mathrm{n}=1690)\end{array}$ & $\begin{array}{l}\text { HAQ } \\
\text { OR }(95 \% \text { CI) } \\
(\mathrm{n}=1688) \\
\end{array}$ \\
\hline Age at disease onset (years) & $0.98(0.97,0.99)$ & $0.98(0.97,0.99)$ & $1.03(1.02,1.05)$ \\
\hline Female gender & $0.32(0.25,0.41)$ & $0.31(0.24,0.39)$ & $2.47(1.76,3.45)$ \\
\hline $\begin{array}{l}\text { Past smoking (vs no smoking) } \\
\text { Current smoking (vs no smoking) }\end{array}$ & $0.58(0.43,0.78)$ & $0.58(0.44,0.77)$ & $1.77(1.20,2.62)$ \\
\hline $\begin{array}{l}\text { Current smoking (vs no smoking) } \\
\text { Positive rheumatoid factor/anti-CCP }\end{array}$ & \begin{tabular}{|l}
$0.56(0.42,0.75)$ \\
$0.83(0.65,1.06)$
\end{tabular} & \begin{tabular}{|l}
$0.66(0.51,0.86)$ \\
$0.82(0.66,1.02)$
\end{tabular} & $\begin{array}{l}1.73(1.19,2.51) \\
1.09(0.79,1.49)\end{array}$ \\
\hline Disease activity score (DAS) & $\begin{array}{ll}- \\
\end{array}$ & $\begin{array}{ll}- \\
\end{array}$ & $3.67(3.41,3.95)$ \\
\hline Presence of baseline erosions & $0.80(0.61,1.04)$ & $0.85(0.67,1.09)$ & $0.63(0.45,0.89)$ \\
\hline Haemoglobin $(\mathrm{g} / \mathrm{dL})$ & $1.03(1.01,1.04)$ & $1.01(1.00,1.03)$ & $1.00(0.98,1.02)$ \\
\hline Recruitment year & $1.09(1.07,1.11)$ & $1.07(1.06,1.09)$ & $1.09(1.07,1.12)$ \\
\hline BMI category $\left(\mathrm{kg} / \mathrm{m}^{2}\right)$ & & & \\
\hline -Underweight & $1.28(0.73,2.26)$ & $1.23(0.75,1.99)$ & $1.83(0.99,3.38)$ \\
\hline -Overweight & $0.97(0.80,1.18)$ & $0.88(0.74,1.05)$ & $0.97(0.77,1.22)$ \\
\hline $\begin{array}{l}\text {-Obese } \\
\text { Refercecateony= nomnal BMI }\end{array}$ & $0.71(0.55,0.93)$ & $0.69(0.55,0.87)$ & $1.63(1.20,2.23)$ \\
\hline
\end{tabular}

Conclusions: The findings support a link between higher BMI and worse clinical outcomes, namely disease activity and functional ability. Obesity was associated with lower levels of both remission and low disease activity states, and of higher disability. The findings highlight the importance of monitoring the patients' weight, screening and targeting obesity as part of routine clinical practice, in order to improve disease outcomes. This work provides clinical insights into the role of $\mathrm{BMI}$ on disease outcomes in RA.

Disclosure of Interest: None declared

DOI: 10.1136/annrheumdis-2017-eular.5510

\section{SAT0672 RISK OF FRAGILITY FRACTURE AMONG PATIENTS WITH PSORIASIS: A POPULATION BASED MATCHED COHORT STUDY FROM THE UNITED KINGDOM}

Z. Paskins ${ }^{1,2}$, R. Whittle ${ }^{1}$, A. Abdul Sultan ${ }^{1}$, S. Muller ${ }^{1}$,

M. Blagojevic-Bucknall ${ }^{1}$, T. Helliwell $^{1}$, S. Hider ${ }^{1,2}$, E. Roddy ${ }^{1,2}$, C. Mallen ${ }^{1}$. ${ }^{1}$ Research Institute for Primary Care and Health Sciences, Keele University, Keele; ${ }^{2}$ Haywood Rheumatology Centre, Haywood Hospital, Burslem, Stoke-on-Trent, United Kingdom

Background: Psoriasis is a common inflammatory skin disease affecting 2-4\% of the population and of these a subset will develop an associated inflammatory arthritis (psoriatic arthritis - PsA). An increased risk of osteoporosis has previously been reported in psoriasis patients but the risk of fracture in patients with both psoriasis and PsA has not been established.

Objectives: To estimate the effect of psoriasis, and PsA, on the risk of fracture using a large electronic primary health care database.

Methods: A matched cohort study was conducted utilizing data from the Clinical Practice Research Datalink, a large UK database of primary care medical records. The exposed population was defined as psoriasis patients aged over 40 years with an incident diagnosis between 1990-2004, who were followed up until 2015. Four unexposed patients were matched to each exposed based on age, sex and general practice. The incidence rate of fracture were calculated as the number of incident diagnoses per 10,000 person-years, stratified by sex. Hazard ratios (HR) and $95 \%$ confidence intervals were estimated using a Cox proportional hazards model to compare the hazard rate between the exposed and unexposed, adjusting for BMI, alcohol consumption, smoking status, Charlson comorbidity index and steroid use. Fracture risk was estimated for patients with both psoriasis and PsA, identified as patients with an incident diagnosis of psoriasis and a diagnosis of PsA between 1990-2004.

Results: 24,219 patients with psoriasis and 94,820 controls were included in the study. The mean age was 59 years at study entry and just over half $(51 \%)$ of the patients were male. The incidence rate of fracture was 58.4 (95\% Cl:55.6-61.3) and 53.1 (51.7-54.5) per 10,000 person-years for the exposed and unexposed, respectively. After adjusting for confounding factors, patients with psoriasis had $12 \%$ increased risk of fracture (HR: 1.12 ; $95 \% \mathrm{Cl}: 1.06-1.19)$ compared to the matched unexposed group. The risk was slightly higher in males (1.22 (1.09- 
1.36)) than females (1.09 (1.03-1.17)). Among those with psoriasis, $4.1 \%$ were also diagnosed with PsA. An increased risk of $45 \%$ was found in those exposed to both psoriasis and PsA compared to the unexposed group (1.45 (1.09-1.94)). Conclusions: This study reports for the first time, an increase in fracture risk in patients with psoriasis. A higher risk was found in males than females and the risk was further increased if the patient also had PsA. These findings suggest that fracture risk assessment needs to be considered for individuals with psoriasis and PsA.

Acknowledgements: This study was funded by the National Institute for Health Research School for Primary Care Research (NIHR SPCR). CDM is funded by the National Institute for Health Research (NIHR) Collaborations for Leadership in Applied Health Research and Care West Midlands, the NIHR School for Primary Care Research and a NIHR Research Professorship in General Practice (NIHR-RP-2014-04-026). TH is funded by an NIHR Clinical Lectureship in General Practice. AAS is funded by an NIHR Postdoctoral Fellowship. The views expressed are those of the author(s) and not necessarily those of the NHS, the NIHR or the Department of Health.

Disclosure of Interest: None declared

DOI: 10.1136/annrheumdis-2017-eular.3379

\section{SAT0673 RISK AGE AND RELATIVE RISK OF CVD IN INFLAMMATORY JOINT DISEASES}

G. Wibetoe ${ }^{1}$, E. Ikdahl ${ }^{1}$, S. Rollefstad ${ }^{1}$, I. Olsen ${ }^{1}$, K. Bergsmark ${ }^{1}$, T. Kvien ${ }^{1}$ A. Salberg ${ }^{2}$, D. Soldal ${ }^{3}$, G. Bakland ${ }^{4}$, A. Lexberg ${ }^{5}$, B.T. Fevang ${ }^{6}$,

H.C. Gulseth ${ }^{7}$, G. Haugeberg ${ }^{8}$, A.G. Semb ${ }^{1} .{ }^{1}$ Diakonhjemmet Hospital, Oslo; ${ }^{2}$ Lillehammer Hospital for Rheumatic Diseases, Lillehammer; ${ }^{3}$ Hospital of Southern Norway, Kristiansand: ${ }^{4}$ University Hospital of Northern Norway, Tromsø; ${ }^{5}$ Vestre Viken Hospital, Drammen; ${ }^{6}$ Haukeland University Hospital, Bergen; ${ }^{7}$ Betanien Hospital, Skien; ${ }^{8}$ Martina Hansens Hospital, Bærum, Norway

Background: Individuals with inflammatory joint diseases (IJD) [rheumatoid arthritis (RA), axial spondyloarthritis (axSpA) and psoriatic arthritis (PsA)] have increased risk of cardiovascular disease (CVD). In the European guidelines for CVD prevention, calculation of relative risk and risk age is advised in patients with low absolute risk of fatal CVD events the next 10 years estimated by the systematic coronary risk evaluation [SCORE] algorithm; the rational being that low absolute risk may conceal high relative risk and risk ages far beyond chronological age. Thus, more patients needing intensive CVD prevention may be identified. Relative risk is a ratio comparing absolute CVD risk in a specific patient to the risk given optimal CVD risk factor levels (CVDRFs). Risk age denotes the age with similar CVD risk and optimal CVDRFs. To this date, no studies have evaluated relative risk and risk age across IJD entities, neither has the agreement between different risk age models been investigated.

Objectives: 1) Estimate relative risk and risk age across IJD entities. 2) Investigate agreement between different risk age models.

Methods: RA/axSpA/PsA patients aged $40 \leq 65$ years with low/moderate 10-year risk of fatal CVD were included from a nationwide quality assurance project implementing CVD risk assessment. Relative risk and cardiovascular risk age was calculated in accordance with risk charts published by the European Society of Cardiology (2016) and Cooney et al (2012), respectively. Vascular age was calculated by matching SCORE to estimated risk ages in accordance with Cuende et al (2010). Four different vascular age estimations were calculated, depending on whether the EULAR 1.5 multiplication factor in RA was applied (mSCORE) and if SCORE version with HDL-C (SCORE-HDL-C) was used: SCORE, SCOREHDL-c, mSCORE and mSCORE-HDL-c. Risk years beyond chronologic age, were calculated. Linear regression models were used to investigate agreement between risk age estimations.

Results: Relative risk was increased in $53 \%$ of all patients and $20 \%$ had three

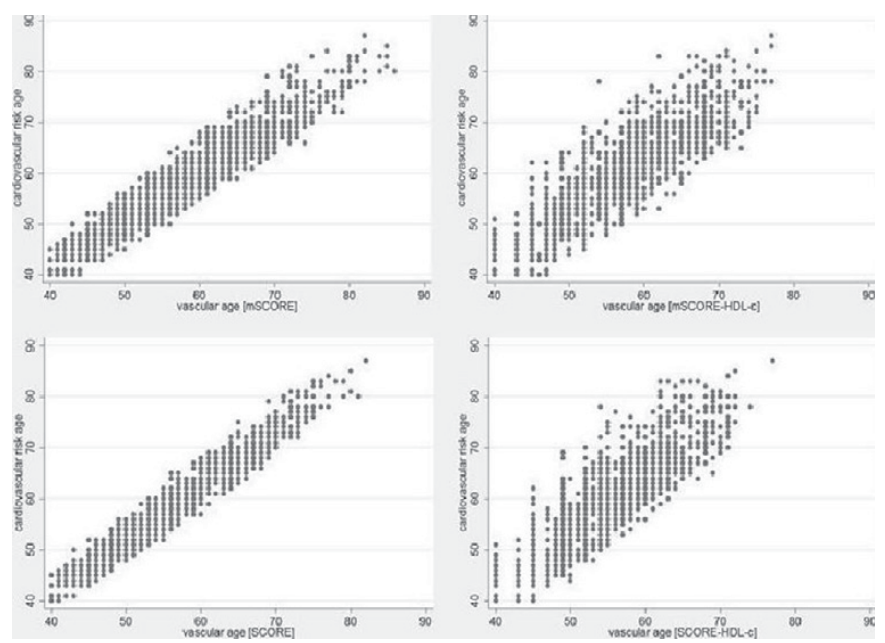

times the risk or higher compared to individuals with optimal CVDRF levels. In total, $42 \%$ and $20 \%$ had a risk age $\geq 5$ years higher than their chronologic age, according to the cardiovascular risk age model and the vascular age model derived from SCORE, respectively. There were minor differences between RA, axSpA and PsA patients in terms of relative risk and risk age. Agreement between cardiovascular risk age and various vascular age models varied (Figure). Discrepancies $\geq 5$ years in estimated risk age were observed in $14-43 \%$ of estimations. The largest observed difference in calculated risk age was 24 years. Similarly, linear regression models yielded a $\mathrm{R}^{2}$ of $0.81-0.96$. Across all models, median difference between risk age and age increased with advancing relative risk. Moreover, several patients had high relative risk despite a risk age close to their chronologic age.

Conclusions: Relative risk and risk age may identify several patients at high need of intensive CVD preventive efforts despite low estimated absolute risk. However, there are considerable discrepancies between risk age models.

Disclosure of Interest: None declared DOI: 10.1136/annrheumdis-2017-eular.2856

\section{SAT0674 EARLY TREATMENT RESPONSE TO CONVENTIONAL DMARD THERAPY IN RHEUMATOID ARTHRITIS IS A BETTER PREDICTOR OF LOW DISEASE ACTIVITY OR TREATMENT ESCALATION AT 12 AND 24 MONTHS THAN AUTOANTIBODIES OR EROSIONS}

A. Richter ${ }^{1}$, A. Strangfeld ${ }^{1}$, P. Herzer ${ }^{2}$, J. Kaufmann ${ }^{3}$, T. Klopsch ${ }^{4}$, S. Zinke ${ }^{5}$, J. Listing ${ }^{1}$, A. Zink ${ }^{1,6} .{ }^{1}$ German Rheumatism Research Center, Berlin; ${ }^{2}$ Scientific Advisory Board, Munich; ${ }^{3}$ Rheumatologist, Ludwigsfelde; ${ }^{4}$ Rheumatologist,

Neubrandenburg; ${ }^{5}$ Rheumatologist; ${ }^{6}$ Charité University Medicine, Berlin, Germany

Background: The EULAR guidelines recommend using the presence of seropositivity or erosions to support treatment decisions. The prognostic value of these factors regarding the primary treatment target in rheumatoid arthritis, remission or low disease activity (LDA), is unclear.

Objectives: To investigate biomarkers, csDMARD treatments and response to treatment regarding their usefulness to predict LDA or the need to escalate treatment within 24 month.

Methods: The control group in RABBIT (Rheumatoid Arthritis: Observation of biologic therapy) comprises $\mathrm{N}=2,228$ patients who were enrolled at treatment start with conventional-synthetic (cs)DMARDs after failure of at least one csDMARD therapy, mostly methotrexate (MTX) monotherapy. We excluded patients with a DAS28-ESR $<3.2$ at enrollment and those with $\geq 2$ csDMARD failures $(n=618)$. 102 patients were excluded due to enrollment less than 12 month prior to closure of the data base (April 30th, 2016). The DAS28-ESR, physical function, age, seropositivity $(\mathrm{RF}+/ \mathrm{ACPA}+)$, comorbidities $(\geq 3$ vs. $<3)$ and the presence of erosions at baseline were evaluated as prognostic factors. Concomitant treatment with glucocorticoids $(\mathrm{mg} / \mathrm{d}$ ) and csDMARDs, response to treatment (36 month after enrollment) were additionally examined. We applied a multinomial generalized-estimating-equation (GEE) model to investigate: (1) achievement of LDA at month 12/24 or (2) treatment escalation (biologic treatment) in year one and two after enrollment.

Results: More than one third of patients $(34.2 \%)$ were treated with a combination of MTX and leflunomide (LEF), $23.6 \%$ with LEF mono, $20.8 \%$ with MTX + hydroxychloroquine (HCQ) or sulfasalazine (SSZ), $16.5 \%$ with MTX mono, and $4.9 \%$ with SSZ mono. We found no major differences across treatment regimens except for patients treated with MTX+HCQ who had a lower DAS28, better physical function and shorter disease duration at treatment start. Significant predictors for achieving LDA were low DAS28 at baseline, improvement in DAS28 within 3-6 month, better physical function and less than 3 comorbidities (table). Escalation to bDMARD therapy was significantly more frequent in younger patients, those with no improvement in DAS28 or concomitant glucocorticoid treatment, and in patients with less than 3 comorbidities. There were no differences between treatments regarding achievement of LDA. However, switching to bDMARDs was

Table: Results of the multinomial GEE-model in odds-ratios (OR) and 95\% confidence interval (CI).

\begin{tabular}{|l|c|c|}
\hline & $\begin{array}{c}\text { Achievement of LDA } \\
\text { at month } 12 \text { or } 24\end{array}$ & $\begin{array}{c}\text { Escalation of Therapy } \\
\text { within } 12 \text { or } 24 \text { month }\end{array}$ \\
\hline Age (per 10 years) & OR $[95 \%$ CI] & OR $[95 \%$ CI] \\
\hline DAS28 improvement $\leq 6$ month (per unit) & $0.97[0.87 ; 1.07]$ & $0.77[0.70 ; 0.86]$ \\
\hline Erosions (Yes vs. No) & $0.95[0.75 ; 1.27]$ & $0.86[0.76 ; 0.97]$ \\
\hline Seropositivity (Yes vs. No) & $0.83[0.67 ; 1.02]$ & $1.16[0.86 ; 1.55]$ \\
\hline DAS at Baseline & $0.67[0.59 ; 0.76]$ & $1.14[1.00 ; 1.17]$ \\
\hline Physical function & $1.13[1.07 ; 1.19]$ & $1.04[0.97 ; 1.11]$ \\
\hline & & \\
\hline$\geq 3$ Comorbidities (vs. $<3$ comorbidities) & $0.73[0.55 ; 0.97]$ & $0.69[0.49 ; 0.98]$ \\
\hline & & \\
\hline Glucocorticoids (per 5mg/d) & $0.96[0.79 ; 1.16]$ & $1.45[1.23 ; 1.71]$ \\
\hline MTX monotherapy (Reference) & & \\
\hline LEF & $0.81[0.58 ; 1.13]$ & $1.55[1.01 ; 2.39]$ \\
\hline MTX+ HCQ | SSZ & $1.20[0.86 ; 1.66]$ & $1.19[0.74 ; 1.90]$ \\
\hline MTX+ LEF & $0.81[0.59 ; 1.11]$ & $1.85[1.24 ; 2.77]$ \\
\hline SSZ & $0.89[0.50 ; 1.59]$ & $1.47[0.74 ; 2.89]$ \\
\hline Year 2 vs. Year 1 & $1.20[0.92 ; 1.56]$ & $1.47[1.11 ; 1.96]$ \\
\hline
\end{tabular}

